\title{
A Co Simulation Of Photovoltaic Power Generation And Human Activity For Smart Building Energy Management And Energy Sharing
}

\author{
Jérémy Albouys-Perrois ${ }^{1,2,3}$, Nicolas Sabouret ${ }^{2}$, Yvon Haradji ${ }^{3}$, \\ Mathieu Schumann ${ }^{3}$, Christian Inard ${ }^{1}$ \\ ${ }^{1}$ LaSIE, CNRS, La Rochelle Université, La Rochelle, France \\ ${ }^{2}$ LIMSI, CNRS, Univ. Paris-Sud, Univ. Paris-Saclay, Orsay, France \\ ${ }^{3}$ EDF R\&D, Palaiseau, France
}

\begin{abstract}
We present a framework and a model for the simulation of collective energy self-consumption strongly coupled to occupant activity in households. The model combines multi-agent simulation of human activity, a dynamic model of building energy including Domestic Hot Water (DHW) and all electrical appliances, and local generation of photovoltaic energy. A cooperative energy exchange policy between households was defined in order to maximise the use of locally generated PV electricity at the neighbourhood scale. In this first implementation, three households A, B and C were simulated, one being the PV energy producer. The DHW model was modified to benefit from PV electricity. Thanks to the energy exchange policy, a total of $83 \%$ of the energy generated by household $\mathrm{A}$ is used locally in the neighbourhood (compared to $38 \%$ when there are no energy exchanges with household B and C). The next step of this study is to add a model of energy storage in several self-consumption configurations.
\end{abstract}

\section{Introduction}

Energy self-consumption consists in generating energy using a local source (photovoltaic panels, windmill, etc.) and consuming part or all of this locally generated energy (Luthander et al. 2015; Matallanas and Monasterio 2011). However, in usual situations, only a small part of this energy can actually be consumed by the producer itself, namely 25 to $40 \%$ according to (Luthander et al. 2015). Several reasons can explain these low figures of selfconsumption.

The building energy needs represent the main part of the electrical energy consumption in the world. According to (Chwieduk 2003; Zimmermann et al. 2012), 57\% of the final energy consumption of the households in Europe is used for space heating. But the periods of energy consumption do not necessarily coincide with the periods of local energy generation. For instance, the need for space heating often occurs while PV panels energy generation is null. In addition, (Bahaj and James 2006) showed that a precise evaluation of electrical energy consumption needs to take human activities into account. But like the heating loads, these energyconsuming activities rarely coincide with the periods of local energy generation.
In this context, one solution to optimise the use of local energy generation is to support energy exchanges between various self-consumer buildings (Tushar et al. 2018; Wu et al. 2015). This can help households to compensate an excess of energy production by using this energy in another household.

Predicting human activities and energy loads of buildings are thus of crucial importance to design practical solutions for efficient energy management in the context of energy sharing. In the present study, both the energy demand of the building and the inhabitant activities are considered.

We used multi-agent systems for simulating human activities coupled with building energy models in order to study energy self-consumption and energy exchanges between households. A multi-agent system can be defined as a set of processes i.e. the agents which exist together, share common resources, and communicate with each other (Ferber 1999). Coupling activity and energy simulation allows to study different exchange configurations and technical solutions which can improve energy self-consumption, such as energy trading (Tushar et al. 2018), energy storage (Braun et al. 2009) or load management (Widén 2014).

The overall goal of our work is to assess the efficiency of several technical and policy solutions for energy exchange, by quantifying the rate of self-consumption at the household and district scale.

This paper focuses on the first implementation of a specific energy exchange policy, and is organised as follows: In the second section, previous works about energy self-consumption, energy exchange and simulation of human activities are presented and discussed. The third section is devoted to presenting the SMACH simulator (Multi-Agent Simulation of Human Behaviour), used for the dynamical simulation of human activities and energy consumption in buildings. Section 4 describes the model of collective energy selfconsumption. Section 5 presents the case study and the results. We then present the conclusions and future work.

\section{Related works}

\section{Multi-Agent Simulation of activity and electrical consumption}

Simulation of human behaviour linked to electrical energy consumption prediction in buildings has been a growing 
centre of interest in the recent years (Ferreri et al. 2017; Kashif et al. 2013; Amouroux et al. 2013). Thus, many studies, based on multi-agent systems (MAS), proposed models to simulate human activity and electrical energy consumption. These models allow a precise simulation of human activity, which is a key factor for electrical energy consumption prediction.

For instance, (Ferreri et al. 2017) used a method based on a psychological model which represents the habits of the inhabitants. This method allows the authors to produce load curves based on the use of electrical appliances. However, the building thermal behaviour is not simulated.

On the contrary (Kashif and al. 2013) proposed a cosimulation where the building and the human activity were simulated. Human behaviour is simulated thanks to Brahms platform (Sierhuis et al. 2007) which supports a fine-grain description of the appliances electrical behaviour. However, this model does not include energy self-consumption and the possibility to simulate exchange between different households.

The approach proposed by the SMACH project (Amouroux et al. 2013) is based on two main components. First, SMACH simulates the human activity by combining fine-grain automated reasoning with statistical behaviour studies (Reynaud et al. 2017). The second component is the multi-zone thermal model of the building, which simulates indoor temperatures and energy consumption. The building is modelled using the BuildSysPro Modelica library, and the SMACH platform and the building energy model are co-simulated using the FMI standard (Plessis et al. 2014). But energy self-consumption and energy exchanges between buildings had not been implemented in these models yet.

\section{Simulation of energy self-consumption}

The various research conducted so far about energy selfconsumption (SC) has focused on improving SC rate of households. The SC rate is usually defined as the ratio of the locally generated energy and energy consumed by the producer during a certain amount of time. (Luthander et al. 2015) reviewed photovoltaic simulations for households and studied various methods used to improve the energy SC rate. They proposed two solutions based on Demand Side Management and storage technologies.

(Braun et al. 2009) studied the impact of using a local battery on the energy self-consumption rate and showed that the amount of local energy consumption can reach $82 \%$ for an individual household. The authors also simulated the energy generation of an empirical PV panels model (based on real data sets) during one-year. However, the authors did not use simulation in order to get consumption data, they used statistical energy consumption profiles for three types of household.

Likewise (Munkhammar et al. 2013) used yearly simulation results to demonstrate that the use of electric vehicles as batteries can improve SC rate by $37 \%$. The method used by the authors for the calculation of energy
SC was a stochastic model based on a Markov-chain and a simple PV model based on solar irradiance data.

However, energy storage systems and Demand Side Management are not the only way to improve the energy $\mathrm{SC}$ rate. With a self-generated energy, it becomes possible for the household to exchange energy with the neighbourhood. The smart grid (SG) technology which can be defined as a network with a bidirectional flow of electricity and information (Farhangi 2010) allows this kind of exchange process. The current research about energy trading focuses on studying algorithms to exchange energy. (Tushar et al. 2018) worked on the ability of algorithms based on a game theory approach to regulate energy exchanges. In addition (Yaagoubi and Mouftah 2017) used a non-cooperative game algorithm to exchange energy between households. They proposed a fair algorithm to trade energy, which means that two households buying local electricity with the same parameters will pay the same price to the producers.

However, the various models previously described do not simulate the link between energy SC and human activity. Most of the time, the human activity models do not include the capacity to simulate the energy SC or energy trading; and the simulation of energy self-consumption is mostly based on a static energy consumption data without any human activity model.

Concerning energy trading configurations related to energy self-consumption, the study of the literature associated to our first surveys led to a first set of categories of exchange policies between households:

- Distribution policy: each household is allowed a fixed rate of energy from each means of production;

- Cooperative policy: the energy produced in the neighbourhood is shared between the households within contractualised rules;

- Competitive policy: the energy will be bought and sold on an energy market where buyers and sellers will interact to exchange energy.

Within this general framework for energy selfconsumption, in which we develop our models and analyses, this paper focuses on one of the identified cooperative policies, as a first implementation for the interaction of self-consumption and human activities in households. In this case study, the use of energy storage systems is not yet considered, but will be added in the following studies, as literature and demonstrators already suggest it has an important impact on self-consumption rate and exchange policies.

\section{Simulation of human activity}

In our work, the simulation of human activity linked to energy consumption is carried out by using the SMACH platform.

In this platform, software agents represent the individuals of the households. Each agent's runtime consists in 
selecting daily tasks to do. The sequence of tasks, chosen by the agent each minute, produces an activity diagram. Moreover, the various activities of the inhabitants are linked to electrical appliances, which consume electricity. As a consequence, the simulator is able to generate load curves associated to the activity diagram (Figure 1 and Figure 3).

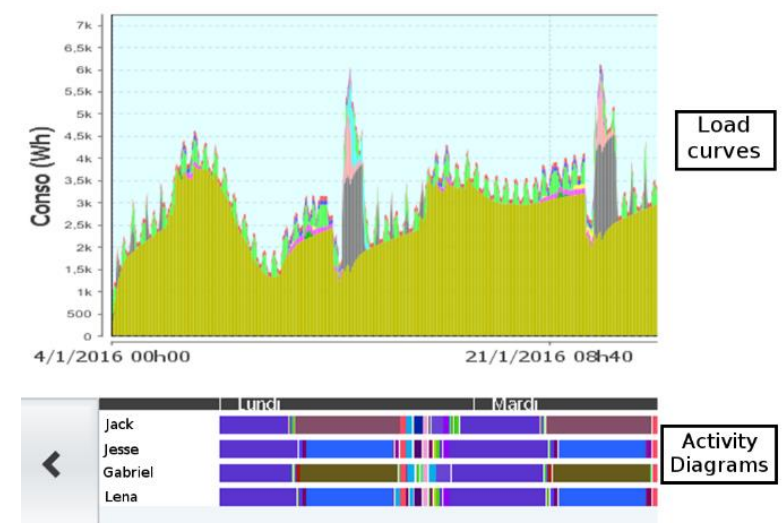

Figure 1: Load curve and activity diagram generated by SMACH

In the next sections, we briefly present the two main dimensions of the simulation, namely the activity model and the agent model:

\section{The activity model}

The initial challenge of the SMACH platform was to produce activity diagrams, on a daily basis, which are realistic representations of human activities throughout the day, and consistent with the different household. SMACH needed also to be able to generate variability in the occupant activities from day to day (Feldman and Pentland 2003), in order to simulate a realistic year.

To achieve this goal, SMACH uses a data-oriented approach based on the results of Time Use Surveys (TUS) (see Reynaud et al., 2017). These surveys consist in 27000 notebooks, on which the 18500 participants wrote their current activity every 10 minutes over a complete week and weekend day. A typology of activities turns these notebooks into a database of human profiles (age, employment, social class, etc) related to the described activities.

The SMACH simulator first generates realistic synthetic households, based on national statistical databases of the population. Each household is composed of one or several agents characterized by their profile (age, socioeconomic class); the building they live in is characterized by its date of construction's thermal regulation, and its appliances. $\mathrm{SMACH}$ also computes the following statistical information from the TUS, for each individual profile and for each possible task the individual can choose to perform:

- The task rhythm, i.e. the average number of repetition of the activity by day or by week.
- The task preferential periods i.e. indicate the periods of the day (or the week) which are favoured to perform the activity.

- The task duration: minimum duration and maximum duration of the activity.

For example, SMACH can extract from the TUS that an upper middle class individual, active, between 20 and 40 years old, living in a household in a specific city would do his shopping once per week, usually around 8:00PM, with a standard deviation of 1 hour and with a duration of 30 minutes with a standard deviation of 15 minutes.

This set of possible tasks is a part of description of the household. This provides a static description of the household's everyday life.

\section{The agent model}

The second part of the SMACH simulator consists in transforming this static description into a dynamic simulation.

At each time step of the simulation, the agents select a task to perform. This selection is made by a mechanism based on dynamic priority levels. The priority levels are modified by parameters which influence the choice of the activity. For example, the current time and the number of previous realisations of the activity, depending on the rhythm and the preferential periods, impact the task selection. The selection mechanism also avoids oscillations between similar tasks, and considers the tasks performed by the others members of the household to satisfy dependency constraints between tasks, and to be able to perform collective activities. The current price of electricity can also lead agents to select less energy consuming tasks during peak-time periods, depending on the household's internal energy policy.

A task dynamically selected from the static tasks pool becomes an action, and is performed by the agent. By realising this action, one agent will also exchange information with the others inhabitant of the household

\section{Evaluation}

The validity of the resulting simulations has been verified by comparing the activity diagrams and the consumption of simulated families with real families (Huraux et al. 2015). The authors used a participatory simulations approach in which participants control the different agents in the household by selecting their activity manually. The participants validate the agent model by comparing the simulated activity diagram with the real activity diagrams obtained through participatory evaluations. The load curves generated by SMACH were also successfully compared to the real measured load curves.

(Reynaud et al. 2017) also validated the activity model and the agent at larger scale. They generated a large amount of activity diagram with SMACH and compared the result with the notebook obtained though the Time Use Surveys. The authors showed that the activity diagrams simulated by SMACH, are similar to the actual 


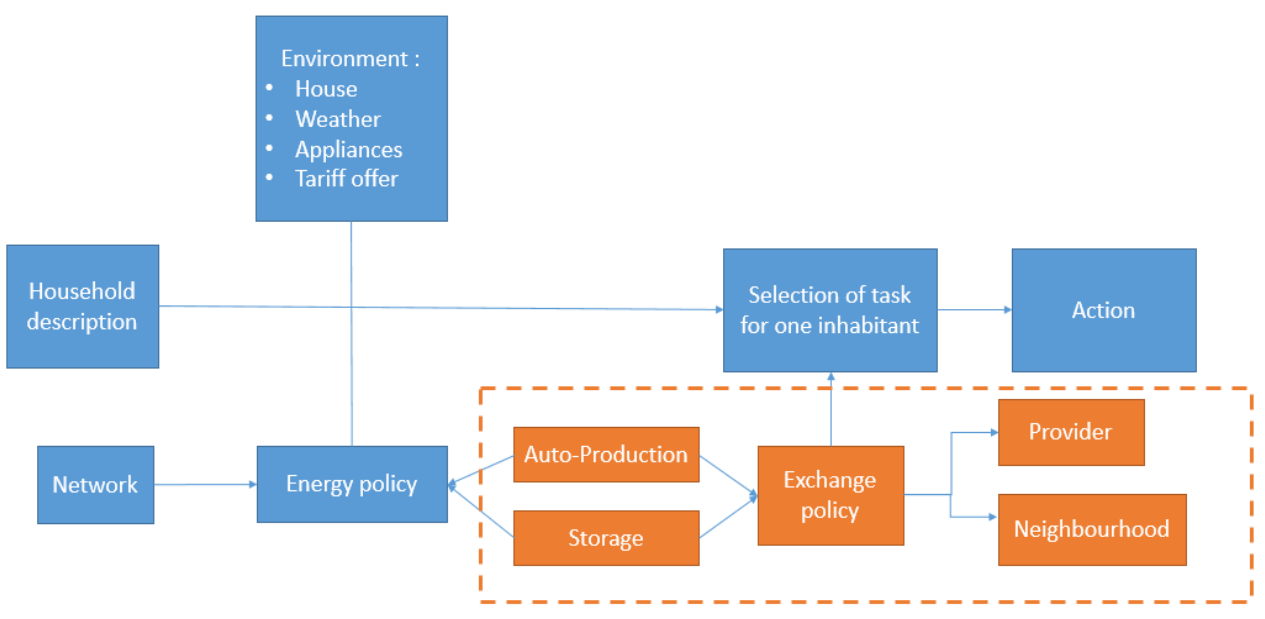

Figure 2: SMACH model with the addition of collective self-consumption

activities from a statistical point of view, with some variability at the individual level. Aggregated load curves produced by SMACH were also compared to aggregated open data load curves from the electric distribution grid, showing a statistical accordance of the platform's results.

\section{Model for collective energy self-consumption}

Our contribution is to integrate energy self-consumption and energy exchange into the SMACH models.

Figure 2 illustrates the general workflow of the SMACH simulator (blue boxes of Figure 2). Starting from the static description of the household, the agent model select a task to perform an action. This action will use electrical appliances. The simulation will compute their consumption and energy cost depending on the current tariff.

The inclusion of the collective energy self-consumption is achieved by adding three new components (orange boxes of the Figure 2). First, we implemented a PV panels simulator. Second, we defined energy self-consumption rules for the appliances. Finally, we defined an energy exchange policy and an energy exchange model for the households.

\section{Adding PV panels to simulate self-consumption}

The building thermal model coupled with SMACH platform was initially developed with the Modelica language and the BuildSysPro library (Plessis et al. 2014). This model is a detailed multi-zone thermal model. For the implementation of the PV panel model, we decided to use the same model library for its simplicity when defining electrical-thermal interfaces.

The model simulates a crystalline silicon PV panels. Its inputs are the weather file, and it computes the generated electrical power (in $\mathrm{kW}$ ) according to the following parameters:

- Surface

- Tilt
- Azimuth

- Cleanliness of the panel

The model of the PV panels is used as a different Fonctional Mock-up Unit (FMU) than the building itself, yet can be interconnected with the building energy model. This allows us to modify the PV panels model and the building thermal model independently, and to pre-process PV generation if needed.

\section{Evolution of the energy consumption model}

We changed the way the various electrical appliances of the household consume the energy produced by the PV panels. Figure 2 shows that the generation of local energy does not affect the agent model as well as the activity diagram. At the stage of the study, only the impact of collective energy self-consumption on the Electric Water Heater (EWH) is studied. This choice was motivated by the fact that this appliance is automatically turned on or off according to the price of the electricity and the temperature of the water. Moreover, excluding electric heating, the EWH is one of the main sources of electrical consumption in the house (Pérez-Lombard et al. 2007).

In our extended model, the EWH will be turned on as soon as the PV panels produce a sufficient amount of energy. If the energy generation is above a threshold, the EWH will use the available energy to heat the water. As an example, an EWH with a nominal power of $2000 \mathrm{~W}$ would use the PV electricity if the PV panel's power exceeds $1000 \mathrm{~W}$. The EWH is given propriety over the other appliances for the consumption of PV electricity. With this operating mode, we guarantee that the $\mathrm{EWH}$ will have the necessary energy to operate.

For the other electrical appliances, first, the energy need of all the appliances is evaluated. Then, we deduce the 


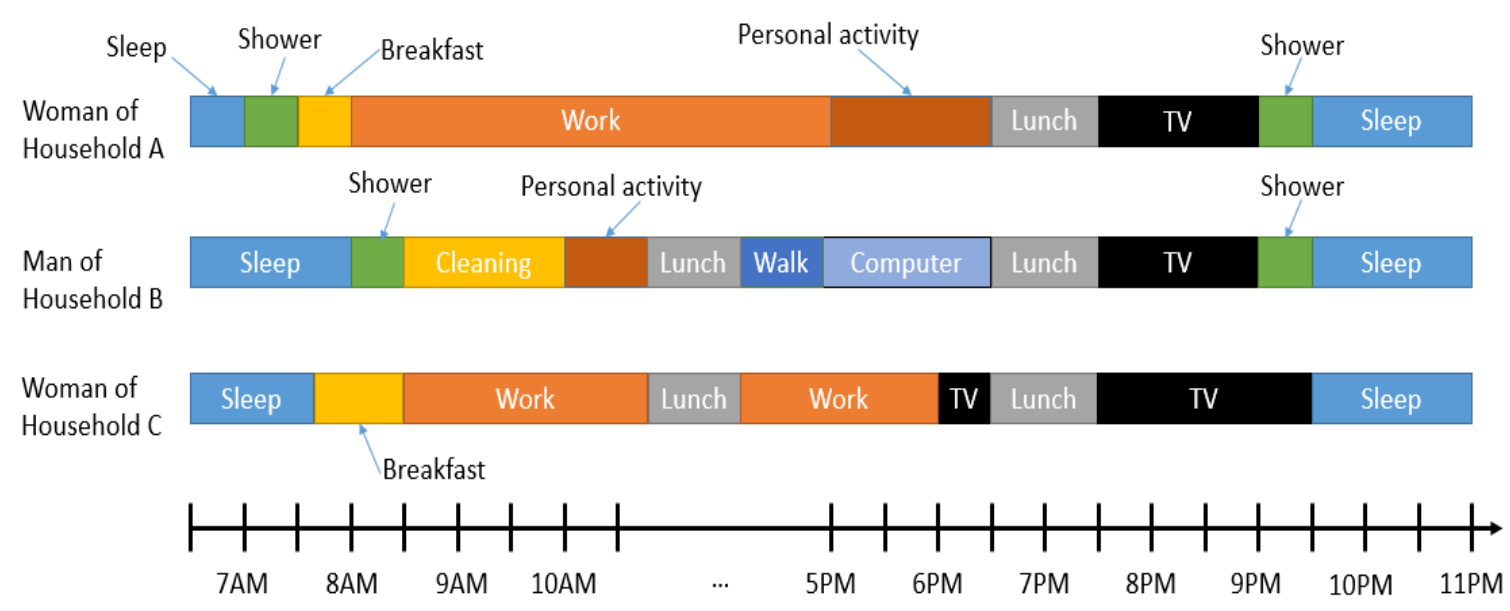

Figure 3: Example of activity diagram of members of household $A, B$ and $C$

remaining available $\mathrm{PV}$ power after the consumption of the EWH.

The EWH can still operate during low-cost energy time period or whenever the water temperature is critically low. In these cases, it will use the electricity from the distribution grid.

\section{Modelling exchanges between buildings}

To implement collective energy self-consumption, the definition of prosumer as defined by (Tushar et al. 2018) is used. Each building in the current simulation can exchange information about the household energy consumption and generation for energy sharing.

Five different states in the process of energy selfconsumption are defined:

1. The prosumer consumes his own production;

2. The prosumer consumes the excess of energy of his neighbours;

3. The prosumer sends the excess of energy from his own production to his neighbours;

4. The prosumer consumes energy from the network;

5. The prosumer sells energy to the network.

The decision of sharing energy depends on the exchange policy agreed by each household. As mentioned in the Related Works, three types of policies namely distribution, cooperative and competitive were identified. The policy affects the amount and price of the energy exchange.

In this first implementation, we focus on the interactions between households with one producer and several consumers, considering states 1, 3 and 4 in the prosumer model. We used an additional model to coordinate the energy exchanges, without simulating the behaviour of an energy trading place in detail.

From the generic framework described above, a cooperative policy in which the producer gives all his excess of energy to his neighbours was adopted, as a first case study. In this model, the consumers will consume all the energy excess they can absorb.

The model consists in two steps. First, the simulation of a time step with only the producer is carried out. He will generate energy with his PV panels and consumes energy according to his activities. Then, he sends a message to all the consumers with the remaining amount of energy.

Second, the same time step for all the consumers is simulated. The consumers use the information about the remaining available energy to support their own energy consumption decisions. They notify to the producer the amount of the energy produced by the PV panels they used during this time step.

All households use the same priority model of energy consumption for the shared PV energy: EWH first, then the other appliances.

\section{Case study and results}

\section{Context of simulation}

The SMACH simulator has the capacity to generate large scale synthetic households and building energy models. The implementation of collective self-consumption at a large scale is part of our current work.

However as a first step, we considered three individual households A, B and C of the same neighbourhood in the city of Nice, France. The three houses are presented in Table 1 and the three families in Table 2.

Two active families (households A and C) and one family of retired inhabitants (household B) are simulated. The households are generated based on the Time Use Survey.

\begin{tabular}{|l|l|l|l|}
\hline & Household A & Household B & Household C \\
\hline Surface & $102 \mathrm{~m}^{2}$ & $110 \mathrm{~m}^{2}$ & $102 \mathrm{~m}^{2}$ \\
\hline Thermal Regulation & 1974 & 1982 & 1974 \\
\hline Electric heater & No & No & No \\
\hline "off-peak" & $11: 00 \mathrm{PM}-7: 00 \mathrm{AM}$ & $10: 30 \mathrm{PM}-6: 30 \mathrm{AM}$ & $11: 00 \mathrm{PM}-7: 00 \mathrm{AM}$ \\
\hline PV panel & Yes & No & No \\
\hline City & Nice & Nice & Nice \\
\hline
\end{tabular}

Table 1: Description of the three houses 
The possible activity diagrams of each household are all different, however households $\mathrm{A}$ and $\mathrm{C}$ present similarities, like the fact that the members of the household go to work during the day (Figure 3).

Household A is an energy producer thanks to its PV panels. Households $\mathrm{B}$ and $\mathrm{C}$ are consumers. For the producer, the PV panels characteristics are the following:

- Area : $20 \mathrm{~m}^{2}$

- Tilt : $30^{\circ}$

- Azimuth : South

The power of the PV panels is $2.5 \mathrm{kWp}$ (kilo Watt-peak). Houses A, B and C have no electrical heating.

Using these models, we assessed the impact of this collective energy self-consumption strategy on the energy consumption of the different families.

We modelled the PV so that the energy generation of household A is sufficient to support a part of the energy consumption of household B. These two families are different in terms of activities. The family of the household A is mainly outdoor during the day when the main part of the local energy is produced whereas for household B the family is indoor during the day

\begin{tabular}{|l|l|l|l|l|l|l|l|l|}
\hline & \multicolumn{3}{|c|}{ Household A } & \multicolumn{3}{c|}{ Household B } & \multicolumn{3}{c|}{ Household C } \\
\hline Situation & Active & Active & Student & Retired & Retired & Active & Active & Student \\
\hline Genre & Woman & Man & Man & Woman & Man & Woman & Man & Man \\
\hline
\end{tabular}

Table 2: Description of the three households

\section{Impact of self-consumption}

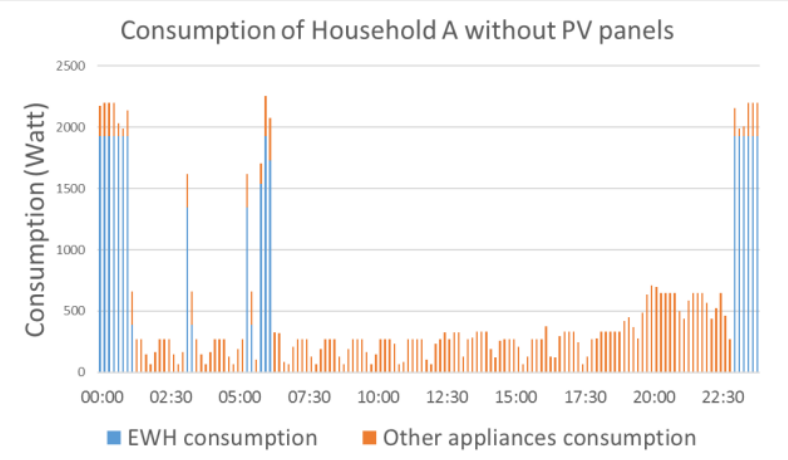

Figure 4: Energy consumption of the EWH and the other appliances without $P V$ panels for the household $A$

Figure 4 shows the energy consumption of the EWH and the other appliances of the household A during one day, without PV panel energy generation. The EWH is only turned on during the off-peak period of the household A, when the price of electricity is cheaper. The total electricity consumption of the appliances is $165 \mathrm{kWh}$ for one month of simulation.

The addition of SC aims at decreasing energy consumption of the EWH from the network, and shifting its energy consumption during the period of energy generation from the PV panels.

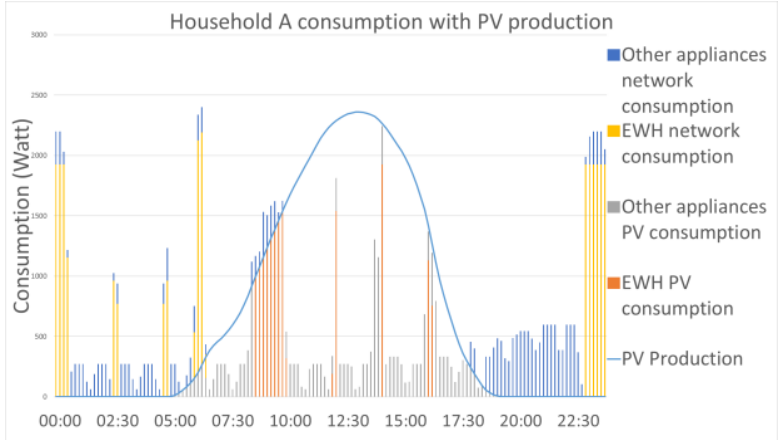

Figure 5: Energy consumption of household A with PV panels energy generation

When there is PV energy generation, Figure shows that a certain amount of the EWH is shifted from night to day. The EWH consumes less energy from the network. The total energy consumption of the appliances in this second simulation is $180 \mathrm{kWh}$ for one month of simulation. Even if the Household is the same, the two activity diagrams of the two simulation which lead to Figure 4 and to Figure 5 are different. This is inherent to the multi-agent simulation which introduces variability from week to week. That is why the total consumption is not the same.

During one month, household A consumes only $40 \%$ of its available PV energy. The energy excess is returned to the network. However, this energy excess could be used as part of exchanges with other households.

\section{Impact of collective energy self-consumption}

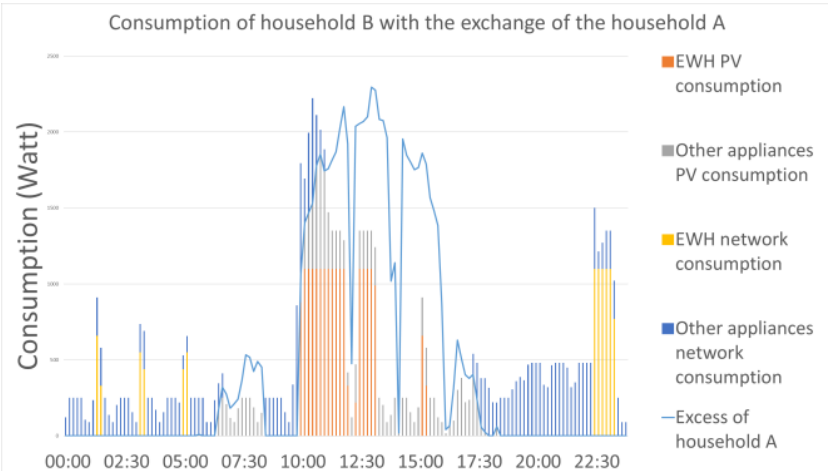

Figure 6: Energy consumption of household B with exchange

Figure 6 shows the consumption of household B while household A exchanges its excess of energy. The energy consumption of household B occurs mainly between $10 \mathrm{AM}$ and 5PM. The EWH energy consumption is the highest during this period. During the night, the EWH is turned on in order to maintain the water set point temperature. The energy consumption in the evening is 
used to balance the water use by the inhabitants for their activities (cooking and washing).

The exchange allows covering $38 \%$ of the energy consumption of the household B. In addition, it provides $62 \%$ of the household A energy to the other houses. So the energy production of one household is sufficient to cover a large part of the energy consumption of the other households thanks to the exchange.

However, $38 \%$ of the household A energy is still unused. Within this cooperative redistribution strategy, we add another house in the exchange, namely household C. This household will consume the excess of energy from household B and more precisely $38 \%$ of the household A energy production.

Figure 7 shows the energy consumption of household C during one day. The profile of the load curve of this house is similar to the load curve of household A. But by looking more closely, only $24 \%$ of the total energy consumption is provided by the energy exchange with household $\mathrm{B}$ and $61 \%$ of this energy is consumed.

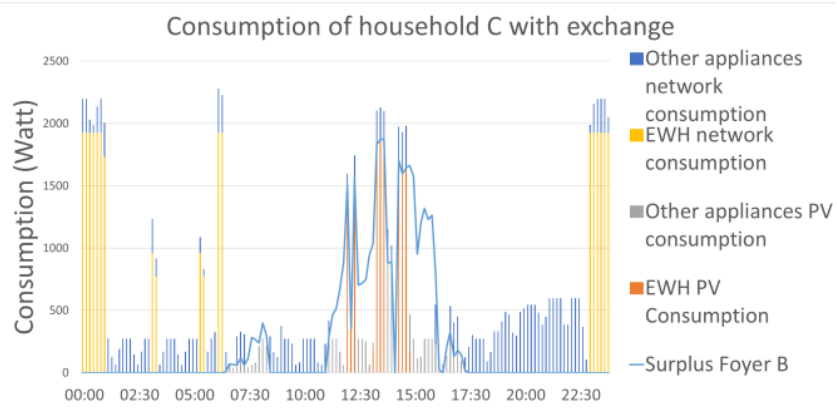

Figure 7: Energy consumption of household $C$ with exchange

The amount of energy received by household $\mathrm{C}$ is low since households A and B consume most of the locally generated electricity.

Moreover, there is always energy that remains unused despite the implementation of the exchange. The gain for household $\mathrm{C}$ is limited due to the fact that members of the family work outside during daytime, hence the importance of taking precisely inhabitants activities into account. A total of $83 \%$ of the energy generated by household $\mathrm{A}$ is used locally in the neighbourhood (compared to $38 \%$ when there are no energy exchanges with household B and C).

\section{Conclusion}

We presented a collective energy self-consumption model coupled with local energy generation by PV panels. A model of energy exchanges between one producer and two consumers was simulated. This model is based on the SMACH platform which simulates realistic human activities and energy consumption of households.
The energy consumption of the EWH calculated for household A remains high even with the PV panels, as $38 \%$ of the energy consumption comes from the distribution network. This energy is consumed mainly during the night while there is no PV generation. In this first use case without energy storage, the amount of PV energy household A consumes is independent of the energy exchange process with household $\mathrm{B}$ and $\mathrm{C}$, as he is the only producer and distributes the remaining energy. Collective energy self-consumption must be used in order to improve the use of locally generated electricity at the district scale.

The general framework and the case study results led to the conclusion that collective energy self-consumption could be relevant for optimizing the use of local energy generation. Moreover, for each energy exchange policy identified in our general framework, energy consumption from local generation or exchange could be increased or optimised through various means, that will be studied in the next steps of our work.

One solution would be to encourage inhabitants to shift some activities or appliance use during the period of energy generation, through information or incentives. However some activities would be difficult to shift, such as fridge operation or lighting. In existing buildings, energy consumption due to TV, computers, cooking and cleaning only represents $10 \%$ of the total energy consumption of the households. In this case, the energy saving brought by the use of PV panels could be low. But the situation could be different for low-energy buildings, for which electrical appliances have a greater importance in the energy balance.

Another solution is the use of an energy storage system in order to store the energy provided by the PV panels. The use of energy storage systems will be include in the study as it will influence energy exchange and consumption. We plan to simulate both collective and individual energy system storage since they impact the energy consumption differently.

We presented a first implementation of our collective selfconsumption framework. The overall goal of this work is to create a model able to study a wide variety of collective energy self-consumption configurations. These configurations will include:

- Various energy exchange policies within a neighbourhood, and between neighbourhoods;

- Electricity prices and their impact on behaviour and energy consumption (the price of locally generated and exchanged electricity, the price of energy storage, and the market price of energy);

- Various equipments related to local energy generation, including those dedicated to energy storage.

The simulation of numerous clusters of households with a collective energy self-consumption policy is also a goal of this work, with several identified strategies, using simplified building models and energy exchange places, where the energy exchange is done at a contractual level. 


\section{References}

Amouroux, É., Huraux, T., Sempé, F., Sabouret, N., \& Haradji, Y. (2013). Simulating human activities to investigate household energy consumption. In Proceedings of the 5th International Conference on Agents and Artificial Intelligence (Vol. 2, pp. 71-80).

Bahaj, A. S., \& James, P. A. B. (2006). Urban energy generation: The added value of photovoltaics in social housing. Renewable and Sustainable Energy Reviews, 11(9),2121-2136.

Braun, M., Büdenbender, K., Magnor, D., \& Jossen, A. (2009). Photovoltaic self-consumption in Germany using Lithium-Ion Storage to increase self-consumed photovoltaic energy. In Proceeedings of the 24th PVSEC (p. 7). Hamburg.

Chwieduk, D. (2003). Towards sustainable-energy buildings. Applied Energy, 76(1-3), 211-217.

Farhangi, H. 2010. "The Path of the Smart Grid." IEEE Power and Energy Magazine 8 (1): 18-28.

Feldman, M. S. and, Pentland, B. T. (2003). Reconceptualizing Organizational Routines as a Source of Flexibility and Change. Administrative Science Quarterly, 48(1), 94.

Ferber, J. (1999). Multi-agent systems : an introduction to distributed artificial intelligence. Addison-Wesley.

Ferreri, E., Salotti, J. M., \& Favier, P. A. (2015). Behavioural approach for the simulation of electrical consumption at the neighbourhood level. International Journal of Simulation: Systems, Science and Technology, 16(3), 12.1-12.8.

Huraux, T., Sabouret, N., Haradji, Y., \& Sempé, F. (2015). Simulations multi-agents de l'activité humaine: application dans le contexte énergétique résidentiel français. Applications Pratiques de l'Intelligence Artificielle (APIA).

Kashif, A., Ploix, S., Dugdale, J., \& Le, X. H. B. (2013). Simulating the dynamics of occupant behaviour for power management in residential buildings. Energy and Buildings, 56, 85-93.

Luthander, R., Widén, J., Nilsson, D., \& Palm, J. (2015). Photovoltaic self-consumption in buildings: A review. Applied Energy, 142, 80-94.

Matallanas, E., \& Monasterio, F. (2011). Analysis of the Self-consumption Possibilities in Small Gridconnected Photovoltaic Systems in Spain. 26th European Photovoltaic Solar Energy Conference and Exhibition, (1), 4619-4624.

Munkhammar, J., Grahn, P., \& Widén, J. (2013). Quantifying self-consumption of on-site photovoltaic power generation in households with electric vehicle home charging. Solar Energy, 97, 208-216.
Pérez-Lombard, L., Ortiz, J., \& Pout, C. (2008). A review on buildings energy consumption information. Energy and Buildings, 40(3), 394-398.

Plessis, G., Amouroux, E., \& Haradji, Y. (2014). Coupling occupant behaviour with a building energy model - A FMI application. In Modelica Conference 2014 (pp. 321-326).

Reynaud, Q., Haradji, Y., Sempé, F., \& Sabouret, N. (2017). Using Time-Use Surveys in Multi Agent Based Simulations of Human Activity. Proceedings of the 9th International Conference on Agents and Artificial Intelligence - Volume 1: ICAART, (Icaart), 67-77..

Sierhuis, M., Clancey, W. J., \& Hoof, R. J. J. Van. (2007). Brahms: a multi-agent modelling environment for simulating work processes and practices. International Journal of Simulation and Process Modelling, 3(3), 134.

Tushar, W., Yuen, C., Mohsenian-Rad, H., Saha, T., Poor, H. V., \& Wood, K. L. (2018). Transforming energy networks via peer-to-peer energy trading: The potential of game-theoretic approaches. IEEE Signal Processing Magazine, 35(4), 90-111.

Widén, J. (2014). Improved photovoltaic selfconsumption with appliance scheduling in 200 singlefamily buildings. Applied Energy, 126, 199-212.

Wu, Y., Tan, X., Qian, L., \& Tsang, D. H. K. (2015). Optimal management of local energy trading in future smart microgrid via pricing. Proceedings - IEEE INFOCOM, 2015-Augus(1), 570-575.

Yaagoubi, N., \& Mouftah, H. T. (2017). Energy trading in the smart grid: A distributed game-theoretic approach. Canadian Journal of Electrical and Computer Engineering, 40(2), 57-65.

Zimmermann, J., Evans, M., Griggs, J., King, N., Harding, L., Roberts, P., Evans, C. (2012). Household Electricity Survey A study of domestic electrical product usage. 